\title{
CONTRIBUTION A L'ÉTUDE DES RUBIS : EXPÉRIENĠES DE R.P.E. DÉTERMINATION DU TEMPS DE RELAXATION SPIN-RÉSEAU A LA TEMPÉRATURE AMBIANTE
}

\author{
Par Serge GLÉMENT, \\ Institut d'Électronique Fondamentale, Laboratoire associé au G.N.R.S. Faculté des Sciences, Orsay.
}

\begin{abstract}
Résumé. - Une généralisation de la méthode de mesure de $T_{1}$ (méthode d'Hervé et Pescia) et une amélioration du spectrographe utilisé pour cette méthode nous ont permis de déterminer la valeur du temps de relaxation électronique spin-réseau dans un échantillon de rubis étudié à la température ambiante.
\end{abstract}

Abstract. - A generalisation of the method of measurement of $T_{1}$ (Hervé and Pescia's method) and an improvment of the spectrometer apparatus used for this method have permitted the determination of the value of the spin-lattice relaxation time in a ruby sample observed at room temperature.

I. Introduction. - Nous nous sommes proposé d'appliquer la méthode d'Hervé et Pescia [1], [2], [3] (méthode permettant de déterminer le temps de relaxation spin-réseau $T_{1}$ d'un échantillon paramagnétique, par utilisation d'un spectrographe hyperfréquence à modulation d'amplitude), à l'étude d'un monocristal de rubis à la température ambiante.

Cette méthode spécialement adaptée à la détermination des $T_{1}$ courts $\left(10^{-6}\right.$ à $\left.10^{-8} \mathrm{~s}\right)$ s'imposait pour une telle étude, les méthodes classiques (limitées à des mesures de $T_{1}$ supérieures à $10^{-6} \mathrm{~s}$ ) ne permettant que des études sur des rubis portés à basse température [4], [5], [6].

Notre mesure exige deux conditions :

a) Sur le plan expérimental. - Adapter le spectrographe que nous avions conçu pour l'étude de poudres polycristallines concentrées [7] à l'étude d'un monocristal qu'il faut orienter avec précision et dont le nombre de centres paramagnétiques est beaucoup plus faible.

b) Sur le plan théorique. - Généraliser la méthode de détermination de $T_{1}$ dont les calculs n'ont été effectués que pour les cas $T_{1}=T_{2}$ et $T_{1} \gg T_{2}$ [1], [2]. Pour le rubis, en effet, des évaluations effectuées sur le $T_{1}$ (extrapolation des mesures à basses températures [8]) et des études de largeur de raies montrent que la valeur du rapport $T_{1} / T_{2}$ peut être de l'ordre de quelques unités, valeur n'entrant pas dans l'application d'un des deux cas mentionnés ci-dessus.
II. Appareillage. - Notre spectrographe utilise un champ hyperfréquence modulé en amplitude (le schéma d'ensemble est représenté sur la fig. 1).

La modulation H.F. est réalisée par l'intermédiaire d'un té simple, par application d'une tension H.F. de l'ordre du volt sur un cristal placé dans un support accordable. A l'aide d'une bobine captrice, d'axe parallèle au champ directeur, et placée à proximité immédiate de l'échantillon, on recueille une tension proportionnelle à la quantité $\frac{\mathrm{d} M_{\mathrm{z}}}{\mathrm{d} t}$. Le signal est amplifié ( $G \simeq 30000$ pour l'amplificateur utilisé), puis détecté linéairement au moyen d'une détection de type Sylvania. Le but de cette linéarisation est de conserver au bruit ses propriétés statistiques initiales et de permettre ainsi l'utilisation d'un dispositif de traitement optimal du signal. Ce traitement est du type détection synchrone : le signal H.F. servant à moduler l'onde U.H.F. est lui-même modulé en amplitude à la fréquence de $1000 \mathrm{~Hz}$. Après la détection linéaire, on obtient un signal à $1000 \mathrm{~Hz}$, d'amplitude proportionnelle au signal cherché, qui est alors détecté de façon synchrone et appliqué à l'entrée Y d'un enregistreur. Une tension proportionnelle à la tension de balayage du champ magnétique étant portée sur l'entrée $\mathrm{X}$, un tel montage nous permet d'enregistrer des spectres dont chaque raie peut être repérée de façon précise.

La cavité hyperfréquence est une cavité travaillant sur le mode $H_{012}$, l'échantillon est placé perpendiculai- 


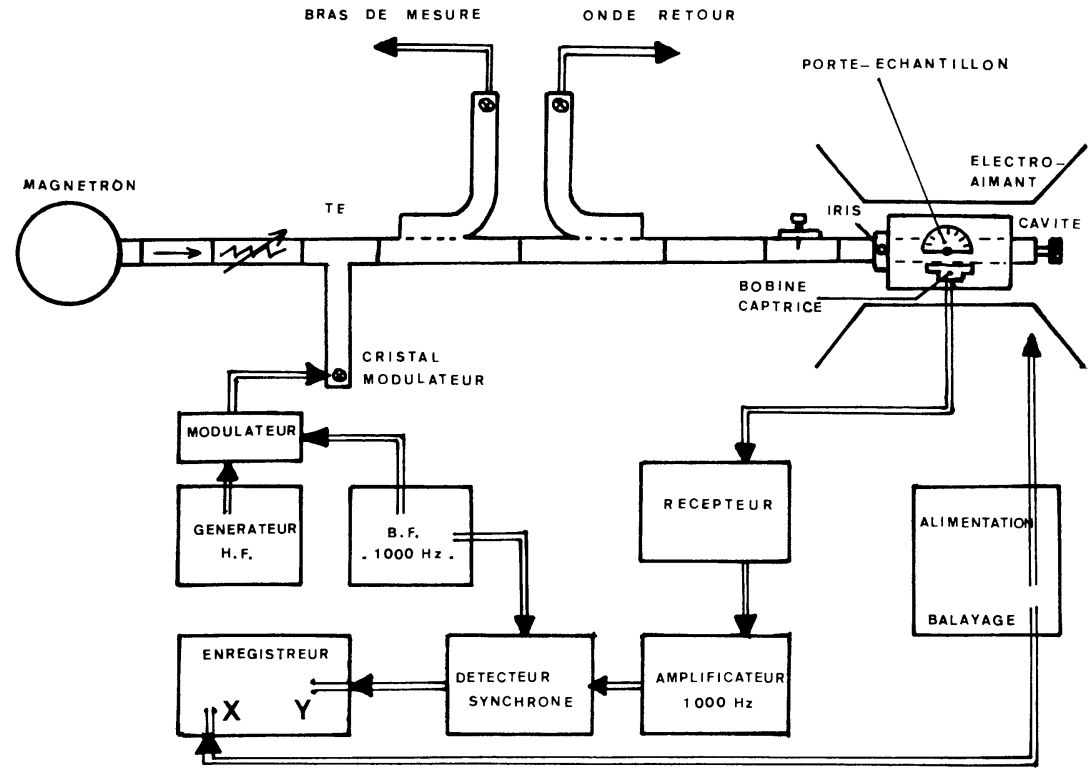

FIG. 1. - Schéma d'ensemble du spectrographe.

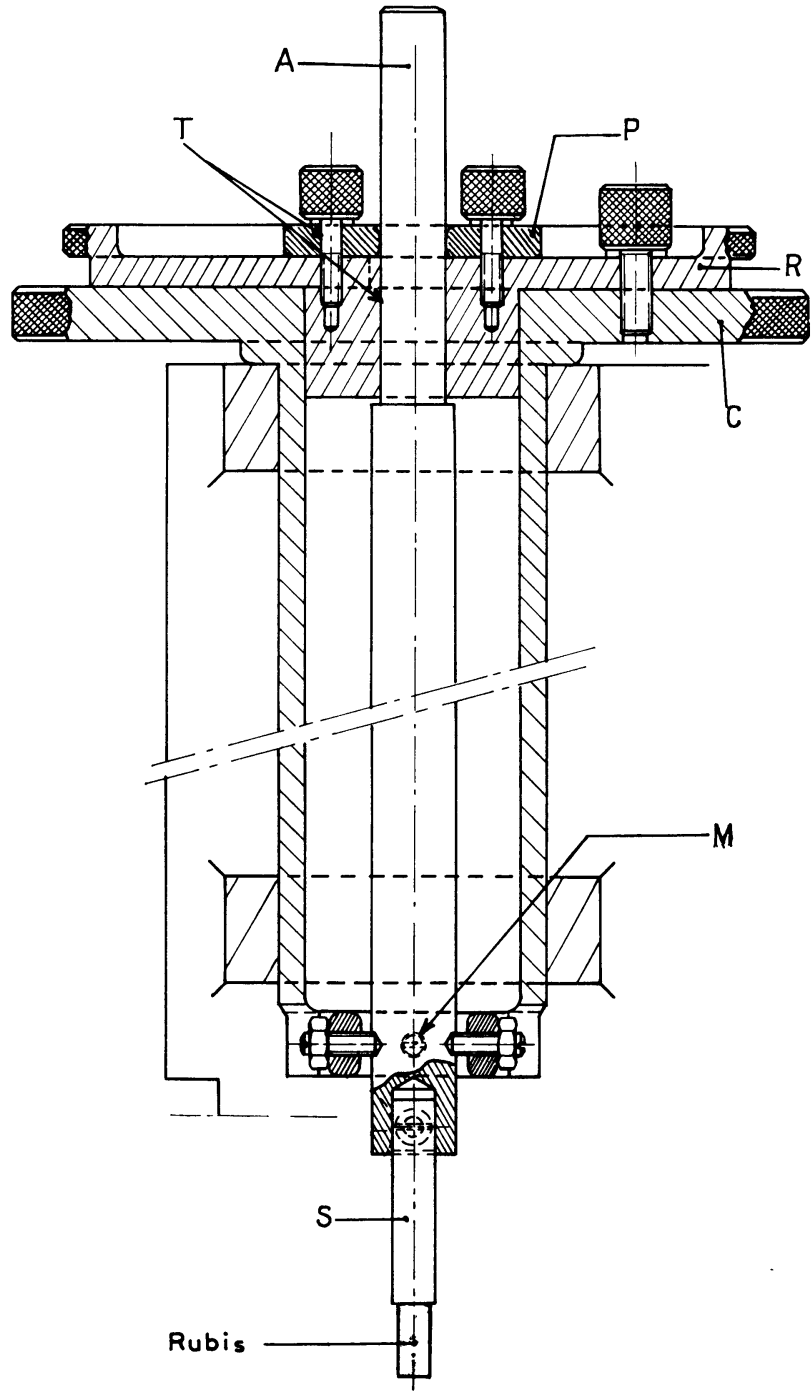

rement au petit côté de la cavité, à un ventre de champ magnétique.

Les monocristaux sont des petits cylindres taillés de telle sorte que l'axe trigonal soit dans le plan d'une section droite. Un porte-échantillon permet de repérer et de faire varier l'angle $\theta$ (angle que fait l'axe trigonal du monocristal avec la direction du champ statique $\mathbf{H}_{\mathbf{0}}$ ).

Ge porte-échantillon ( fig. 2) est muni d'un système compensateur qui permet d'éliminer une petite erreur d'orientation pouvant être due au cristal lui-même ou au montage mécanique qui le supporte.

III. Détermination de $T_{1}$ dans le cas général. Dans nos expériences, l'échantillon est soumis à un champ directeur $\mathbf{H}_{\mathbf{0}}$ (définissant l'axe $\mathrm{Oz}$ ) et à un champ hyperfréquence perpendiculaire, modulé en amplitude. On détecte alors la variation de l'aimantation le long du champ directeur. Le calcul du signal peut être effectué en supposant le mouvement de l'aimantation $\mathbf{M}$ régi par l'équation vectorielle de Bloch :

$$
\frac{\mathrm{d} \mathbf{M}}{\mathrm{d} t}=\gamma \mathbf{M}_{\wedge}\left(\mathbf{H}_{\mathbf{0}}+\mathbf{H}_{1}\right)-\frac{\mathbf{M}_{\mathbf{I I}}}{T_{\mathbf{1}}}-\frac{\mathbf{M}_{\perp}}{T_{\mathbf{2}}}
$$

- $\gamma$ est le rapport gyromagnétique de l'électron, - $\mathbf{H}_{0}$ le champ statique,

- $H_{1}$ le champ hyperfréquence :

$$
\mathbf{H}_{\mathbf{1}}=2 \hat{\mathbf{H}}_{\mathbf{1}}(1+m \cos \Omega t)
$$

FIG. 2. - Porte-échantillon : A, axe de commande du réglage. - $T$, trous oblongs. - $P$, plaquette de blocage. - $\mathrm{R}$, plaquette auxiliaire permettant un déplacement dans une direction. - $\mathrm{C}$, commande de rotation de l'ensemble. - M, montage à point fixe. $\mathrm{S}$, support de quartz. 
- $\mathbf{M}_{11}$ et $\mathbf{M}_{\perp}$ sont respectivement les composantes de l'aimantation parallèle et perpendiculaire au champ statique; $T_{1}$ et $T_{2}$ étant les temps de relaxation correspondants.

L'équation (1) se résout par une méthode de perturbation.

En se plaçant à la résonance, le signal recueilli par la bobine a pour expressions [1], [2], [3] :

$S(\Omega)=S_{0} \times \frac{\Omega T_{1}\left\{1+\left(\frac{\Omega T_{2}}{2}\right)^{2}\right\}^{1 / 2}}{\left\{\left[1+a-\Omega^{2} T_{1} T_{2}\right]^{2}+\Omega^{2}\left(T_{1}+T_{2}\right)^{2}\right\}^{1 / 2}}$

- $\Omega / 2 \pi$ représente la fréquence de modulation,

- $a$ est le facteur de saturation, sa valeur est : $a=\gamma^{2} H_{1}^{2} \cdot T_{1} \cdot T_{2}$.

En nous plaçant dans des conditions de faible saturation $(a \ll 1)$, nous allons étudier les propriétés essentielles de la famille de courbes représentatives de la fonction $Z_{\mathrm{p}}(X)$, fonction qui a pour expression :

$$
Z_{\mathrm{p}}(X)=\frac{S(\Omega)}{S_{0}}=\frac{X\left(1+\frac{p^{2} X^{2}}{4}\right)^{1 / 2}}{\left[\left(1+X^{2}\right)\left(1+p^{2} X^{2}\right)\right]^{1 / 2}}
$$

avec

$$
X=\Omega T_{1} \quad \text { et } \quad p=\frac{T_{2}}{T_{1}} .
$$

Pour toute expérience de résonance paramagnétique électronique, le paramètre $p$ satisfait à la condition : $0<p \leqslant 1$.

Une telle fonction $Z_{\mathrm{p}}(X)$ (représentée fig. 3) possède une partie croissante, passe par une valeur maximale, puis décroît asymptotiquement vers la valeur $1 / 2$.

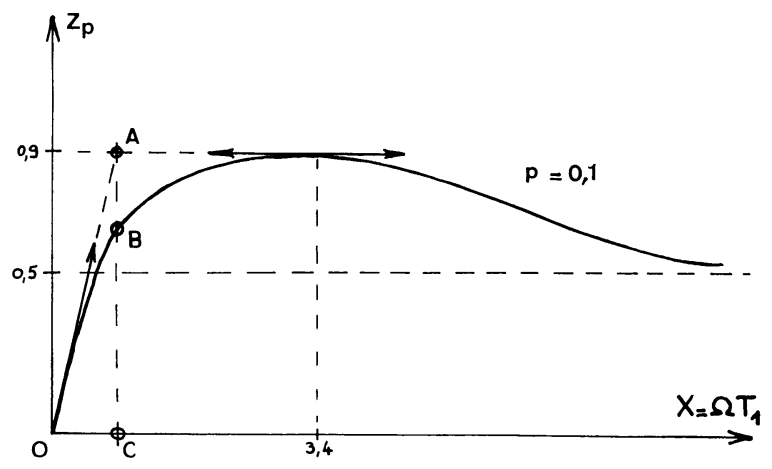

Fig. 3. - Exemple de courbe représentative de la fonction $Z_{\mathrm{p}}(X)$.

Pour $p=0$, le maximum est rejeté à l'infini, la fonction est uniformément croissante et tend vers 1 .

- La tangente à l'origine est la même pour toutes les courbes, la pente prend la valeur 1 .

- La valeur maximale de la fonction a pour expression :

$$
\left(Z_{\mathrm{p}}\right)_{\mathrm{Max} .}=\frac{\sqrt{3}}{2} \cdot \frac{p+\left(12-3 p^{2}\right)^{\mathbf{1 / 2}}}{3+p\left(12-3 p^{2}\right)^{\mathbf{1 / 2}}} .
$$

Nous déterminons la valeur de $T_{1}$ au moyen de deux grandeurs considérées, d'une part sur la courbe expérimentale, et d'autre part sur la courbe théorique.

Ces deux grandeurs sont :

- Le rapport $r(p)=\frac{\mathrm{AC}}{\mathrm{BC}}$ (voir fig. 3).

- L'abscisse $X_{0}(p)$ du point d'intersection de la tangente à l'origine avec la tangente au maximum $\left(X_{0}=\overline{\mathrm{OG}}\right)$.

La valeur du rapport $r(p)$ est donnée par l'expression :

$$
r(p)=\left\{\frac{\left(4+A^{2}\right)\left(4+p^{2} A^{2}\right)}{16+p^{2} A^{2}}\right\}^{1 / 2}
$$

avec

$$
A=\sqrt{3} \cdot \frac{p+\left(12-3 p^{2}\right)^{1 / 2}}{3+p\left(12-3 p^{2}\right)^{1 / 2}} .
$$

L'expression de $X_{0}(p)$ est identique à celle de $\left(Z_{\mathrm{p}}\right)_{\text {Max. }}$.

En donnant à $p$ différentes valeurs de l'intervalle $(0,1)$, nous pouvons déterminer point par point les fonctions $r(p)$ et $X_{0}(p)$.

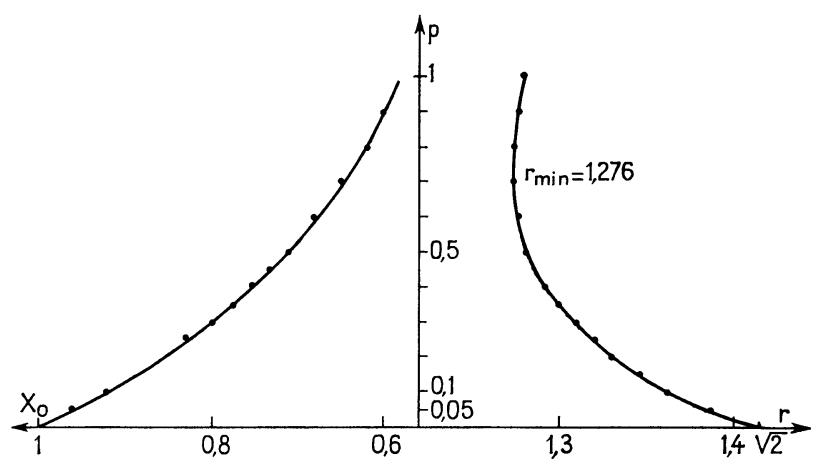

FIG. 4. - Courbes représentatives des fonctions $r(p)$ et $X_{0}(p)$.

Les calculs ont été effectués par utilisation d'une machine électronique du type CAB 500 et ont permis de tracer les courbes représentées sur la figure 4. La détermination d'un $T_{1}$ est alors aisée.

A partir d'une courbe expérimentale $S(\Omega)$, la construction des deux tangentes nous détermine une valeur $r_{\text {exp., }}$ le point d'intersection correspondant à l'abscisse $\Omega_{\text {exp. }}$. A partir de la valeur $r_{\text {exp. }}$ et par utilisation de nos « courbes étalon », nous déduisons immédiatement la valeur de $p$ et celle de $X_{0}$. Le temps de relaxation spin-réseau $T_{1}$ est alors donné par le rapport :

$$
T_{1}=\frac{X_{0}}{\Omega_{\text {exp. }}} .
$$

La connaissance du paramètre $p$ nous permet de déterminer le temps de relaxation spin-spin :

$$
T_{\mathbf{2}}=p T_{\mathbf{1}} \text {. }
$$

L'intérêt de cette méthode est de pouvoir déduire d'une seule courbe $S(\Omega)$ les deux grandeurs $T_{1}$ et $T_{2}$. 
IV. Résultats expérimentaux. - 1. SPECTRE DU RUBIS. - Dans un premier stade, pour un rubis donné, nous nous sommes proposé d'enregistrer de nombreux spectres relatifs à différentes orientations de l'échantillon, ce qui nous a permis de dresser une « carte » du

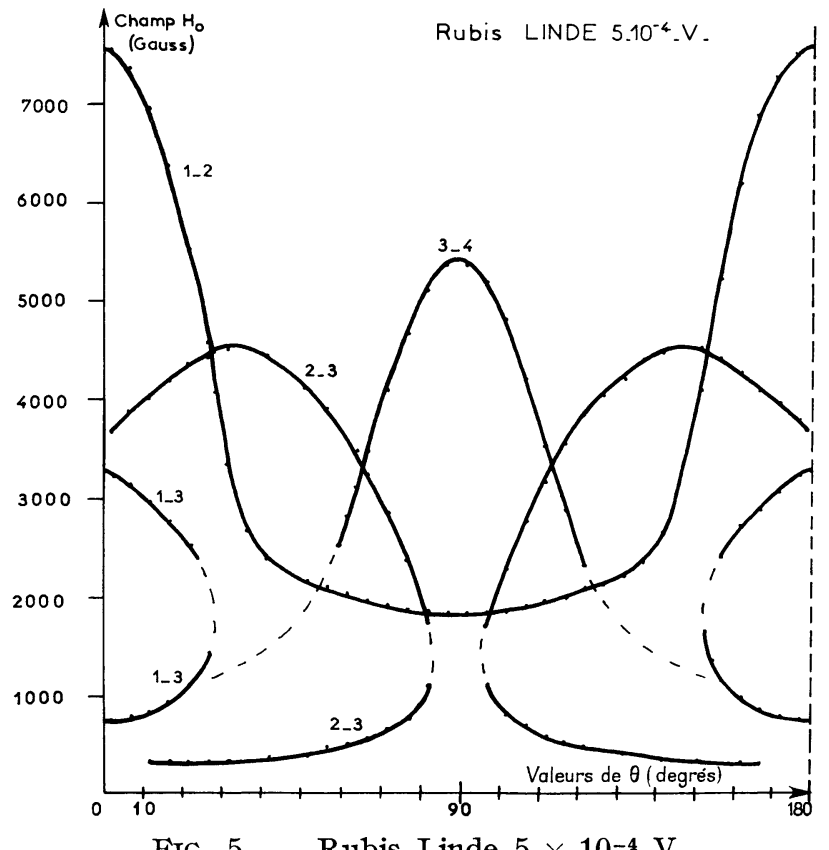

FIG. 5. - Rubis Linde $5 \times 10^{-4} \mathrm{~V}$.

Transition 2-3 champ faible

Transition 1-2

Transition 2-3 champ fort

$$
\begin{aligned}
& p=0,15 \\
& p=0,3 \\
& p=0,15
\end{aligned}
$$

déplacement des raies en fonction de l'orientation du monocristal. L'intérêt d'une telle « carte » est de nous permettre de pouvoir définir à tout instant les paramètres relatifs à une quelconque expérience. Il est, en effet, impératif, pour des mesures de $T_{1}$, de pouvoir déterminer à partir d'un spectre quelconque la valeur de l'angle que fait l'axe trigonal du cristal avec le champ statique de manière à l'ajuster ensuite à la valeur choisie.

La figure 5 représente la « carte » du déplacement des raies d'un échantillon de rubis Linde, la fréquence de la source hyperfréquence étant dans ce cas voisine de $9200 \mathrm{MHz}$. Cette " carte » est analogue à celles présentées pour des fréquences différentes par d'autres auteurs [5], [9].

2. Détermination DU Temps DE RELAXation SPINRÉSEAU. - Nos mesures ont été effectuées sur un monocristal de rubis dont la croissance a été obtenue par le procédé Verneuil, le dopage de notre échantillon étant de $5 \times 10^{-4}$. (Rappelons que ce dopage correspond en fait au rapport des ions $\mathrm{Cr}^{3+}$ vis-à-vis des atomes d'Al. de la matrice alumine.) Le cristal a été orienté de telle sorte que l'angle $\theta$ prenne la valeur de $50^{\circ}$, la température de l'expérience étant la température ambiante.

Les résultats déduits de nos courbes expérimentales et de notre " courbe étalon » sont consignés dans le tableau ci-dessous :

$$
\begin{array}{ll}
T_{1}=3,3 \times 10^{-7} \mathrm{~s} & T_{2}=5 \times 10^{-8} \mathrm{~s} \\
T_{1}=2 \times 10^{-7} \mathrm{~s} & T_{2}=6 \times 10^{-8} \mathrm{~s} \\
T_{1}=3,3 \times 10^{-7} \mathrm{~s} & T_{2}=5 \times 10^{-8} \mathrm{~s}
\end{array}
$$

Une telle méthode s'est alors montrée féconde dans son application à l'étude d'un monocristal de rubis.

Remerciements. - Cette étude a été effectuée au Laboratoire de l'Institut d'Électronique Fondamentale de la Faculté d'Orsay sous la direction de M. le Professeur P. Grivet en collaboration avec le Centre de Recherches de la Compagnie Générale d'Électricité qui a bien voulu mettre à notre disposition un certain nombre d'échantillons.

Je remercie également M. J. N. Aubrun pour les précieux conseils dont il a su me faire profiter.

Manuscrit reçu le 16 décembre 1966. rapport $T_{2} / T_{1}$.

\section{BIBLIOGRAPHIE}

[1] Hervví et Pescia, C. R. Acad. Sci., 1960, 251, 665.

[2] Hervví et Pescia, C. R. Acad. Sci., 1962, 255, 2926.

[3] Pescia, Thèse, Arc. Sc., 13e série, 1964, 9.

[4] Pace-Sampson et Thorp, Proc. Phys. Soc., 1960, 76, 697.

[5] Standley et Vaughan, Phys. Rev., 1965, 139, 1275.
[6] Manenkov et Danileiko, Sov. Phys. JetP, 1965, 257.

[7] Clément et Pescia, C. R. Acad. Sci., 1965, 260, 2143.

[8] VAN DER ZIEL et BloEMbERgen, Phys. Rev., 1965, 138, 1287.

[9] SchulZ-DU-Bors, Bell System, 1959, 38, 271.

[10] GILL et ELLIOT, Adv. in Quantum Electronics, $1961,399$. 